\title{
Die Auswirkungen der Stornier- und Umbuchfunktion in Truck Appointment Systemen
}

\author{
Katharina Beck ${ }^{1}$, Ann-Kathrin Lange ${ }^{1 *}$, Carlos Jahn ${ }^{1}$ \\ ${ }^{1}$ Institut für Maritime Logistik, Technische Universität Hamburg, Am Schwarzenberg-Campus 4 (D), 21073 Hamburg; \\ *ann-kathrin.lange@tuhh.de
}

\begin{abstract}
Größere Containerschiffe führen zu höheren Umschlagmengen je Anlauf und damit zu Peaks im Zu- und Ablauf der Containertransporte per Lkw. Um die Ankunftszeitverteilungskurve abzuflachen, haben viele Terminals in den vergangenen Jahren sogenannte Truck Appointment Systeme (TAS) eingeführt. In diesen Systemen buchen Lkw Zeitfenster für die Aufnahme oder Abgabe von Containern. Bei Abweichungen vom geplanten Transportablauf stehen teilweise unterschiedliche Flexibilitätsoptionen zur Zeitfensteranpassung zur Verfügung. Neben dem Tauschen von vorhandenen Slots können die Zeitfenster je nach Verfügbarkeit auch storniert und umgebucht werden. Die Studie untersucht die Auswirkungen der Nutzung der Flexibilitätsoptionen Tauschen, Umbuchen und Stornieren auf die KPI von Transportunternehmen unterschiedlicher Größe und bei verschiedenen Verkehrssituationen im Hafengebiet. Um die komplexen Prozesse realitätsnah abbilden zu können, wird ein diskretes ereignisorientiertes Simulationsmodell (DES) erstellt.
\end{abstract}

\section{Einführung}

Im Jahr 2018 wurden weltweit 784 Mio. TEU (Twentyfoot Equivalent Unit) umgeschlagen [1]. Immer größer werdende Schiffe und neu formierte Reedereiallianzen, welche ihre Services mit geringerer Frequenz und dafür mit mehr Containern pro Anlauf planen, erhöhen die Komplexität der intermodalen Transportketten [2]. Die Zunahme der je Anlauf umgeschlagenen Container führen zu Peaks bei der Containeranlieferung und -abholung mittels Lkw und übersteigen die Gate- und Yardbearbeitungskapazitäten der Terminals [3]. Zur Verbesserung des Ankunftszeitmanagements von Lkw haben weltweit viele Terminals TAS eingeführt, deren Ziele u. a. eine bessere Koordinierung des Containerflusses und die Reduzierung von Staus und Wartezeiten sind [2]. Bei TAS werden die Öffnungszeiten der Terminals in Zeitslots unterteilt. Jedem Zeitslot wird eine Slotkapazität zugewiesen, die unter anderem abhängig vom zur Verfügung stehenden Handlingequipment ist. Die Lkw können die zu ihren Wünschen passenden Slots buchen, bis die voreingestellten Kapazitäten ausgeschöpft sind [4]. Das gebuchte Zeitfenster gibt den Zeitkorridor vor, in dem der
Lkw am Terminal ankommen muss [5]. Die implementierten TAS sind unterschiedlich ausgestaltet und bieten den Transportunternehmen teilweise verschiedene Flexibilitätsoptionen, um auf Störungen im Transportablauf reagieren zu können [6]. Die vorliegende Arbeit soll die Fragestellung klären, welche Auswirkungen die Möglichkeit des Stornierens und Umbuchens auch in Kombination mit der Möglichkeit des Tauschens von Zeitfenstern in TAS auf unterschiedliche Leistungsindikatoren wie Auftragserfüllungsquote, Wartezeit oder Pünktlichkeit eines Transportunternehmens besitzt.

\section{Stand der Forschung}

Bisher gibt es wenig Studien über die Auswirkungen von TAS auf die Produktivität von Transportunternehmen oder Evaluierungen von TAS hinsichtlich der Auswirkungen verschiedener Zeitfensterbreiten, Strafen oder Flexibilitätsoptionen auf das Gesamtsystem [7,8].

Das zu lösende Problem der vorliegenden Arbeit lässt sich wie folgt beschreiben: Im Hafenbereich muss eine bestimmte Anzahl an Containern von einem Fuhrunternehmen transportiert werden. Die identischen Lkw (homogener Fuhrpark) starten und enden an einem Depot und transportieren im Tagesverlauf Container zwischen Depots, Kunden und Servicestationen (z. B. Terminals oder andere intermodale Anlagen). Bei der Lösung des Problems sind die durch die Kunden und Servicestationen vorgegebenen Zeitfenster für die Abgabe und Aufnahme des Containers, die auf einen Container je Transportauftrag begrenzte Fahrzeugkapazität und die maximal zulässige Lenkzeit des Fahrers zu beachten $[9,10]$. Diese Tourenplanungsprobleme und Vehicle Routing Probleme sind NP-schwer und exakte Algorithmen sind nur bei kleinen Problemstellungen möglich. Die Anzahl der Schritte, um alle durchführbaren Touren zu ermitteln, steigt exponentiell mir der Problemgröße an und macht die exakte Lösung des Problems häufig unmöglich, weshalb auf Heuristiken und Methaheuristiken zurückgegriffen wird $[11,8]$.

In der Literatur werden unterschiedlich ausgestaltete TAS betrachtet. Yi et al. [12] untersuchen ebenso wie 
Chen et al. [13] ein TAS, bei welchem Slots in einem iterativen Prozess auf Grundlage der voraussichtlichen Wartezeit gebucht werden. Torkjazi et al. [14] untersuchen ein Centralized TAS, bei welchem die Slots von einer übergeordneten unabhängigen Plattform an die Lkw vergeben werden, die die angefragten Slotzeiten mit einbezieht. Ein kollaboratives TAS wird von Azab et al. [15] untersucht, bei welchem der Terminal die Abfertigungsprozesse simuliert, um die Durchlaufzeiten der angefragten Zeitfenster zu ermitteln. Die Fuhrunternehmen passen ihre Slotzeiten entsprechend an. Phan et al. [16] betrachten einen Verhandlungsprozess über Slots, um die Summe aller Wartezeiten, den Stau und die Kosten für Verspätungen zu reduzieren. Eine Kollaboration von Transportunternehmen in einem Hafen mit TAS wird von Schulte et al. [5] beleuchtet. Zehendner et al. [17] ermitteln die optimalen Kapazitäten der Zeitfenster in TAS in Terminals, die zusätzlich auch Schiffe und Bahnen abfertigen. Wie sich unterschiedliche Verteilungen der Zeitfensterkapazitäten und -breiten im TAS auf die Fuhrunternehmen auswirken, wird von Namboothiri et al. [18] untersucht. Shiri et al. [19] erweitern das Problem von [18] und beziehen zusätzliche Restriktionen wie Zeitfenster beim Kunden oder die Leercontainerallokation mit ein.

Huynh et al. [7] stellten fest, dass Studien, die TAS optimieren, meistens mathematische Programmierungen verwenden. Studien, die die Auswirkungen von TAS Parametern untersuchen nutzen eher Simulations- oder Warteschlangenmodelle. Dabei eignen sich speziell DES, um bspw. Lkw-Abläufe auf dem Terminalgelände sowie den Betrieb des Terminalequipments detailliert darzustellen und mit unterschiedlichen Szenarien zu testen [20].

So nutzen Azab et al. [15] DES für die Bestimmung der Durchlaufzeiten der Lkw, Do et al. [21] für die Vorhersage von Wartezeiten und Fahrstrecke des Handlingequipments, Huynh [22] und Huynh et al. [23] für die Auswirkungen der gewählten Zeitfensterkapazitäten auf die Terminalprozesse und Lkw, Ramírez-Nafarrate et al. [2] für den Gate Betrieb, van Asperen et al. [24] für die Auswirkungen von TAS auf die Performance der Containerstapelregeln und Speer [25] für die Optimierung von automatischen Lagerkransystemen. Schulte et al. [5] verwenden DES zur Simulation einer Kollaboration zwischen Fuhrunternehmen in einem Hafen mit TAS.

DES zur Untersuchung der Auswirkungen von TAS auf die Tourendurchführung von Transportunternehmen finden sich nach Kenntnis der Autoren nur in Lange et al. [26].

\section{Aufbau der Simulationsstudie}

Das DES wurde mit Tecnomatix Plant Simulation Version 15 erstellt und basiert auf Daten verschiedener Unternehmen des Hamburger Hafens. Dabei werden neben den vier großen Containerterminals 18 weitere Knoten wie Leercontainerdepots, Packbetriebe, sonstige Logistikknoten und das Fahrzeugdepot betrachtet.

Die Simulationsstudie soll untersuchen, ob sich die Möglichkeiten der flexiblen Stornierung und Umbuchung von Zeitfenstern in TAS positiv auf relevante Kennzahlen von Transportunternehmen, wie z. B. Anzahl der pro Tag durchgeführten Aufträge, Wartezeit vor den Knoten, Auftragsabbrüche oder Pünktlichkeit der Ankunft, auswirken. Dabei wird geprüft, ob eine besonders gute oder eine besonders schlechte Verkehrssituation Auswirkungen auf die Häufigkeit der Nutzung der Flexibilitätsoptionen besitzt und ob die positiven Effekte der Flexibilitätsoptionen unterschiedlich groß sind. Es werden unterschiedliche Unternehmensgrößen und verschiedene Schichtmodelle betrachtet, um Aussagen über deren Auswirkungen auf die Unternehmenskennzahlen auch in Kombination mit den Flexibilitätsoptionen treffen zu können. Es wird erwartet, dass kleine Unternehmen öfter umbuchen müssen, da größere Unternehmen mehr Tauschmöglichkeiten zur Anpassung ihrer Transporte besitzen und dass eine Kombination der Funktionen Umbuchen und Tauschen die positiven Effekte verstärkt.

Die Studie erweitert die Arbeit von Lange et al. [26] um die Möglichkeiten der Nutzung der Stornier- und Umbuchfunktion und verwendet folgende an [26] angelehnte Annahmen:

- Das Simulationsmodell ist auf den Bereich des Hamburger Hafens begrenzt.

- Ein Simulationslauf umfasst ein bis zwei Schichten. Alle erforderlichen Buchungen im TAS werden zu Beginn jedes Laufes getätigt.

- Im Tagesverlauf gehen keine neuen Transportanfragen ein.

- Zeitfensterbuchungen sind nur an den Containerterminals notwendig.

- Neben den Containerterminals haben nur vier Knoten ebenfalls 24/7 geöffnet.

- Transportaufträge gebuchter Zeitfenster können (auch kurzfristig) getauscht und bis vor Zeitfensterbeginn storniert und umgebucht werden, ein Hinzubuchen ist nicht möglich.

- Ein Transportauftrag besteht aus dem Abholen und Ausliefern eines Containers.

- Die Routenzeiten sind durch gesetzliche Lenkpausen und Schichtzeiten begrenzt. 
Je nach Ankunftszeit am Terminal werden die Lkw unterschiedlichen Prioritätsgruppen zugeordnet, die sich auf die Art der Abfertigung beziehen. Die Zeitfensterbreite der Priorität 1-Abfertigung beträgt eine Stunde, wobei es eine Toleranz von je 30 Minuten vor und nach dem Zeitfenster gibt. Dadurch beträgt die Zeitfensterbreite, in denen ein Lkw mit der höchsten Priorität 1 abgefertigt wird, 120 Minuten. Sollte das Fuhrunternehmen erkennen, dass es sein Zeitfenster nicht einhalten kann, bietet das TAS den Fuhrunternehmen Flexibilitätsoptionen, um auf Veränderungen im Transportablauf reagieren und die Slots anpassen zu können. Bestehende Slots können storniert und umgebucht oder getauscht werden. Das Stornieren und Umbuchen ist kurzfristig möglich und beeinflusst nur die Slotzeit des betreffenden Transportes. Beim Tauschen werden die Slotzeiten von zwei Transporten miteinander getauscht. Tauschen ist unabhängig von der Slotverfügbarkeit des Terminals immer möglich, eine Umbuchung kann nur dann erfolgen, wenn das Zeitfenster, in welches umgebucht werden soll, noch freie Kapazitäten besitzt. Stornierungen nach Zeitfensterbeginn werden als No-Shows gezählt.

Mittels der durch Lange et al. [26] implementierten VBA wird die Auftragsliste für den untersuchten Tag in drei Schritten erstellt. Auf Grundlage der Struktur des Transportaufkommens werden Aufträge zufällig auf einzelne Verbindungen verteilt und in der Transportmatrix vermerkt. Für den Slotbuchungsprozess werden die Kapazitäten außerhalb der Betriebszeiten auf null gesetzt und die Kapazitäten in den verbliebenen Zeitfenstern je Terminal begrenzt. Die Kapazität je Zeitfenster berechnet sich aus der Multiplikation des einstellbaren Faktors mit der Anzahl der insgesamt benötigten Slotbuchungen und wird durch die Betriebszeit der Fuhrunternehmen dividiert. Die Zeitfensterbuchung erfolgt mit Zufallszahlen und in zufälliger Reihenfolge. Bei Aufträgen, die zwei Buchungen benötigen, wird versucht, das gleiche oder eines der beiden nachfolgenden Zeitfenster für die Senke zu buchen. Zusätzlich werden die Öffnungszeiten der Knoten und die Betriebszeiten des Fuhrunternehmens berücksichtigt.

Wie bei [26] erfolgt die dynamische Fahrzeugführung im Simulationsmodell und die Aufträge werden mit einer fahrzeuginitiierten Auftragszuweisung vergeben. Dabei werden neben der Dringlichkeit des Auftrages, welche von den Slotzeiten sowie etwaig vorhandenen Schließzeiten abhängt, auch die Fahrzeit zur Quelle des Folgeauftrages berücksichtigt. Bei der Vergabe des Auftrages wird nach Möglichkeit bereits ein passender Folgeauftrag vorgemerkt. Mögliche Tauschoptionen werden vor der Vergabe des nächsten durchzuführenden Auftrages geprüft. Ob eine Umbuchung für den aktuellen und auch für einen eventuell bereits eingeplanten Folgeauftrag notwendig ist, wird erst nach der Auftragszuweisung untersucht. Sollte eine Umbuchung notwendig sein, so muss geprüft werden, ob noch Slots im gewünschten Zeitfenster verfügbar sind. Die Slotverfügbarkeit ändert sich im Zeitverlauf und ist abhängig von der Entfernung des Zeitpunktes der Buchung zum gewünschten Slottermin. Diese Dynamik bei der Slotverfügbarkeit wird durch die Erzeugung einer gleichverteilten Zufallszahl abgebildet.

Durch die unsicheren bzw. teilweise unvollständigen Informationen muss die Planung der Zeitfenster und des Transportablaufes unter Umständen mit den aktualisierten Informationen in einer rollierenden Planung modifiziert bzw. wiederholt werden [27]. Zu den unsicheren bzw. teilweise unvollständigen Informationen zählen z. B. die Slotverfügbarkeit im Tagesverlauf, die Auslastung der Terminals, die Möglichkeit einer Abfertigung auch außerhalb der gebuchten Slotzeit oder die reale Fahrzeit.

Um den Transport nachverfolgen zu können, wurden im Modell die Ereignisse Ankunft und Abfahrt am Knoten als sogenannte Meldepunkte definiert. Bei Eintritt dieses Ereignisses übermittelt der Lkw seine Position und die aktuelle Uhrzeit. Bei Ankunft an der Quelle wird die voraussichtliche Verweildauer, bestehend aus Warteund Bearbeitungszeit, am Knoten zur Ermittlung der voraussichtlichen Abfahrtzeit (Estimated Time of Departure (ETD)) sowie die Fahrzeit zur Senke prognostiziert. Die damit ermittelte voraussichtliche Ankunftszeit (Estimated Time of Arrival, (ETA)) an der Senke, die hier ein Containerterminal darstellt, wird mit der vorhandenen Slotzeit verglichen.

Sollte der Lkw nicht innerhalb des Zeitkorridors seines Slots ankommen, so wird versucht, den Slot umzubuchen. Für alle bekannten Folgeknoten wird das Verfahren wiederholt. Ergebnisse etwaiger Umbuchungen der Vorgängerknoten werden bei der Planung berücksichtigt. Nach Abschluss des aktuellen Auftrages wird ein Folgeauftrag zugewiesen, der in der Regel dem bereits reservierten Folgeauftrag entspricht. Sollten sich spontan Änderungen hinsichtlich der Dringlichkeit eines durchzuführenden Auftrages ergeben, so kann der Disponent auch einen anderen Folgeauftrag zuweisen.

Mit der Google Distance Matrix API werden die tageszeitabhängigen Fahrzeitmatrizen erstellt, wobei diese neben der normalen Fahrzeit auch die Fahrzeiten für eine sehr gute und sehr schlechte Verkehrssituation ermitteln. Dadurch sollen die Auswirkungen unterschiedlicher Verkehrssituationen und die daraus resultierende Notwendigkeit einer Anpassung der Zeitfenster als zusätzlicher 
Einflussfaktor der Transportdurchführung untersucht werden. Die Fahrzeiten werden im Modell vereinfacht als Verweilzeiten auf den jeweiligen Bausteinen betrachtet. Das dynamische Modell bezieht den Zeitaspekt und die Veränderungen der Fahrzeiten mit ein.

\section{Experimente}

Das Simulationsmodell untersucht drei verschiedene Unternehmensgrößen, von der die Anzahl der durchzuführenden Aufträge abhängt. Das kleine Unternehmen arbeitet im Ein-Schicht-Betrieb von 6 bis 15 Uhr, das große im Zwei-Schicht Betrieb von 4 bis 13 Uhr und von 13 bis 22 Uhr. Das mittlere Unternehmen nutzt beide Schichtmodelle. Die Fahrzeiten können für eine normale, gute und schlechte Verkehrslage berechnet werden.

Tabelle 1 zeigt die untersuchten Fuhrunternehmensgrößen, die Anzahl der Schichten sowie Fahrer und Aufträge. Bei zwei Schichten werden jeweils 50 \% der Fahrer pro Schicht eingesetzt.

\begin{tabular}{ccccc}
\hline Nr. & $\begin{array}{c}\text { Unterneh- } \\
\text { mensgröße }\end{array}$ & $\begin{array}{c}\text { Anzahl } \\
\text { Schichten }\end{array}$ & $\begin{array}{c}\text { Anzahl } \\
\text { Fahrer }\end{array}$ & $\begin{array}{c}\text { Anzahl } \\
\text { Aufträge }\end{array}$ \\
\hline $\mathbf{1}$ & Klein & 1 & 12 & 65 \\
\hline $\mathbf{2}$ & Mittel & 1 & 36 & 195 \\
\hline $\mathbf{3}$ & Mittel & 2 & 36 & 195 \\
\hline $\mathbf{4}$ & Groß & 2 & 60 & 325 \\
\hline
\end{tabular}

Tabelle 1: Experimentplan mit ausgewählten Parametern

Es werden vier verschiedene Module untersucht (Tabelle 2). Neben einem Grundmodell werden eine Strategie, die nur Tauschen ermöglicht, und eine Strategie, die nur Umbuchen ermöglicht, betrachtet. Ebenso wird eine Kombination aus Tauschen und Umbuchen untersucht. Je Modul werden 100 Simulationsläufe durchgeführt.

\begin{tabular}{cl}
\hline Modul & Name des Moduls \\
\hline $\mathbf{1}$ & Grundmodell mit Folgeauftrag \\
\hline $\mathbf{2}$ & Tauschen mit Schwerpunkt Leerfahrt minimieren \\
\hline $\mathbf{3}$ & Umbuchmodul \\
\hline $\mathbf{4}$ & Gesamtmodul mit Umbuchen und Tauschen \\
\hline
\end{tabular}

Tabelle 2: Übersicht verschiedene Module und mit erlaubten Umbuchoptionen

\section{Ergebnisauswertung}

Tabelle 3 zeigt die Unterschiede bei der Auftragserfüllungsquote (AEQ) des Grundmodells (Modul 1) der drei Unternehmensgrößen und zwei Schichtmodelle.

\begin{tabular}{ccccc}
\hline & klein & mittel & mittel & groß \\
\hline $\begin{array}{c}\text { Fuhrunterneh- } \\
\text { men Nr. }\end{array}$ & 1 & 2 & 3 & 4 \\
\hline $\begin{array}{c}\text { AEQ normaler } \\
\text { Verkehr }\end{array}$ & $91,9 \%$ & $93,0 \%$ & $88,3 \%$ & $88,9 \%$ \\
\hline $\begin{array}{c}\text { AEQ guter } \\
\text { Verkehr }\end{array}$ & $96,2 \%$ & $97,1 \%$ & $92,7 \%$ & $93,5 \%$ \\
\hline $\begin{array}{c}\text { AEQ schlechter } \\
\text { Verkehr }\end{array}$ & $87,6 \%$ & $89,0 \%$ & $83,4 \%$ & $84,6 \%$ \\
\hline
\end{tabular}

Tabelle 3: Vergleich verschiedener Auftragserfüllungsquoten im Grundmodell bei verschiedenen Verkehrssituationen

Es ist erkennbar, dass die AEQ bei einer Schicht (Unternehmen 1 und 2) besser ist als bei zwei Schichten (3 und 4), wobei die Unterschiede zwischen Unternehmen unterschiedlicher Größe aber gleichem Schichtmodell bei normalem Verkehr gering sind. Bei einer sehr guten Verkehrssituation steigt die AEQ um 4,1 \% bis 4,6 \% an. Die positiven Effekte fallen für Unternehmen mit zwei Schichten größer aus. Das große Unternehmen profitiert am meisten vom guten Verkehr. Eine schlechte Verkehrssituation reduziert die AEQ um 4,0 \% bis 4,9\%, wobei die negativen Auswirkungen am größten für das mittlere Unternehmen mit zwei Schichten sind.

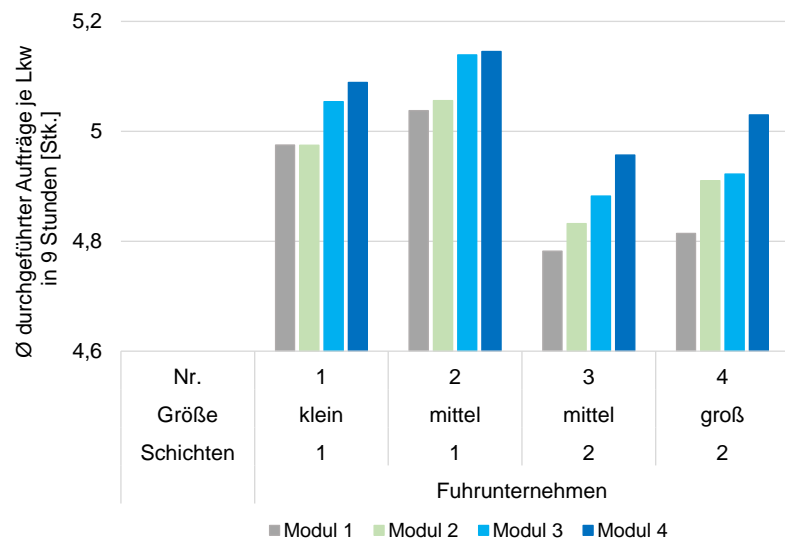

Abbildung 1: Anzahl durchschnittlich durchgeführte Aufträge je Lkw in 9 Stunden

Alle Flexibilitätsoptionen erhöhen die Anzahl der in 9 Stunden durchgeführten Aufträge im Vergleich zum Grundmodell. Modul 3 (nur Umbuchen) führt zu besseren Ergebnissen als Tauschen mit Leerfahrt minimieren (Abbildung 1). Das Gesamtmodul führt stets zu besseren Ergebnissen als Module mit nur einer Flexibilitätsoption. 
Die Anzahl der durchgeführten Aufträge pro Lkw von Unternehmen mit einer Schicht ist größer als bei zwei Schichten. Bei gleichem Schichtmodell führt das größere Unternehmen mehr Aufträge durch.

Eine gute Verkehrssituation hat positive Auswirkungen auf die Anzahl der durchgeführten Aufträge. Bei einer schlechten Verkehrssituation sinkt die Anzahl der durchgeführten Aufträge bei allen Unternehmen, wobei die Auswirkungen auf Unternehmen mit zwei Schichten größer sind als auf Unternehmen mit einer Schicht.

Es wird deutlich, dass die Anzahl der Schichten bei der Anzahl der durchgeführten Aufträge entscheidender ist als die Größe des Unternehmens. Unternehmen mit einer Schicht können innerhalb ihrer Schicht mehr Aufträge durchführen. Grund hierfür ist die Struktur des Transportaufkommens, bei der $60 \%$ einen und $20 \%$ aller Aufträge zwei Logistikknoten beinhalten. Diese Logistikknoten haben größtenteils begrenzte Öffnungszeiten, die teilweise außerhalb der Schichtzeiten der zweiten Schicht liegen. Die Aufträge können daher nur in der ersten Schicht durchgeführt werden.

Bei zwei Schichten sind die Auswirkungen der Änderungen der Verkehrssituation in Prozent größer, da die Lkw von Transportunternehmen mit zwei Schichten neben der Morgenspitze des Verkehrs von 06:00 bis 07:00 Uhr auch während der Nachmittagsspitze von 15:00 bis 16:00 Uhr im Einsatz sind.

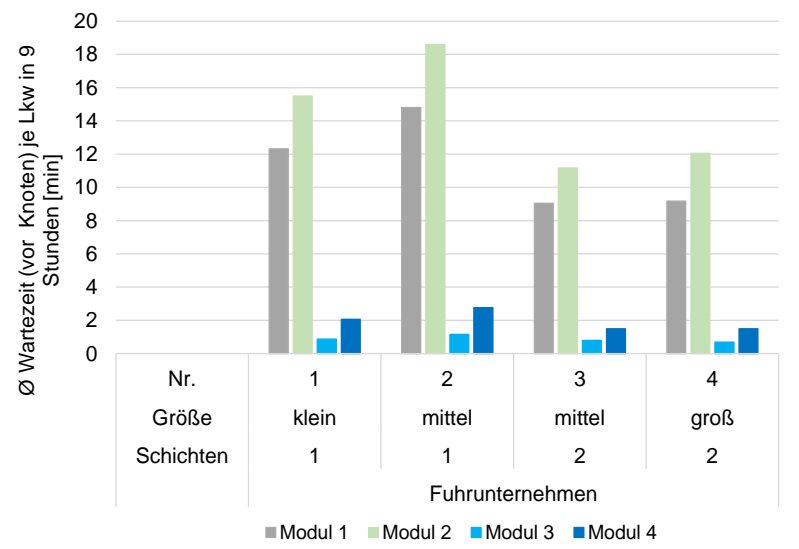

Abbildung 2: Durchschnittliche Wartezeit vor Knoten je Lkw in 9 Stunden

Die Wartezeit vor dem Knoten fällt nur an, wenn der Lkw am Terminal außerhalb seiner Slotzeit ankommt oder den Logistikknoten vor der Öffnung erreicht (Abbildung 2). Unabhängig von der Unternehmensgröße ist bei allen Modulen (3 und 4), die ein Umbuchen erlauben, die Wartezeit vor den Knoten am geringsten.

Beim Modul, welches nur das Tauschen erlaubt (Modul 2), ist die Wartezeit vor den Knoten größer als im Grundmodell. Wenn nur Umbuchen erlaubt ist (Modul 3) ist die Wartezeit vor den Knoten am geringsten und insgesamt warten Unternehmen mit zwei Schichten kürzer als Unternehmen mit einer Schicht. Bei einer guten Verkehrssituation sind die Wartezeiten vor den Knoten länger, bei einer schlechten Verkehrssituation sind sie kürzer. Die Zunahme bei der guten Verkehrssituation beträgt bei einer Schicht rund 5 bis 10 Minuten bei den Modulen 1 und 2 sowie 3 bis 6 Minuten bei zwei Schichten. Bei den Umbuchmodulen beträgt die Zunahme maximal eine Minute. Bei einer schlechten Verkehrssituation nimmt die Wartezeit um 2 bis 5 Minuten bei den Modulen 1 und 2 bei einer Schicht und maximal 2 Minuten bei zwei Schichten ab. Die Wartezeiten bei den Umbuchmodulen sind annähernd gleich.

Die Wartezeit vor den Knoten ist, unabhängig von der Unternehmensgröße, bei allen Modulen, die ein Umbuchen erlauben (3 und 4), am geringsten und mit Wartezeiten unter vier Minuten innerhalb von neun Stunden zu vernachlässigen. Dies deckt sich mit dem Ziel der Erreichung des Terminals während der Priorität 1-Abfertigung, welches mit der Umbuchung verfolgt wird. Die Wartezeit vor den Knoten bei den anderen Modulen ist abhängig von der Anzahl der Schichten, wobei die Wartezeiten bei zwei Schichten geringer sind als bei einer Schicht. Bei einer guten Verkehrssituation sind die Wartezeiten insgesamt höher, da die Lkw schneller als geplant den Knoten erreichen. Da sowohl die initiale Slotbuchung als auch die Auftragszuweisung die erwarteten Fahrzeiten berücksichtigen, sind die Wartezeiten vor den Knoten bei Modulen ohne erlaubte Umbuchung höher. Bei einer schlechten Verkehrssituation sind die Wartezeiten vor den Knoten geringer. Da die Auftragszuweisung einen gewissen Puffer berücksichtigt und die Knoten damit tendenziell eher vor als nach dem Slottermin erreicht werden, fallen trotz schlechter Verkehrssituation noch Wartezeiten an.

Eine weitere wichtige Kennzahl ist der Anteil der Auftragsabbrüche bei der Durchführung aufgrund einer Ankunft außerhalb der Öffnungszeiten an den Logistikknoten oder außerhalb der erlaubten Abfertigungstoleranz an den Terminals (Abbildung 3). Beim Grundmodell werden anteilig die meisten Aufträge abgebrochen (2,5\% bis 3,5\%), wobei das große Unternehmen die meisten Auftragsabbrüche vorzuweisen hat. Bei einer Schicht führt das Tauschen (Modul 2) zu mehr Abbrüchen als das Umbuchen (Modul 3), bei zwei Schichten ist es umgekehrt. Eine gute Verkehrssituation führt insgesamt $\mathrm{zu}$ weniger Auftragsabbrüchen, eine schlechte zu mehr. 


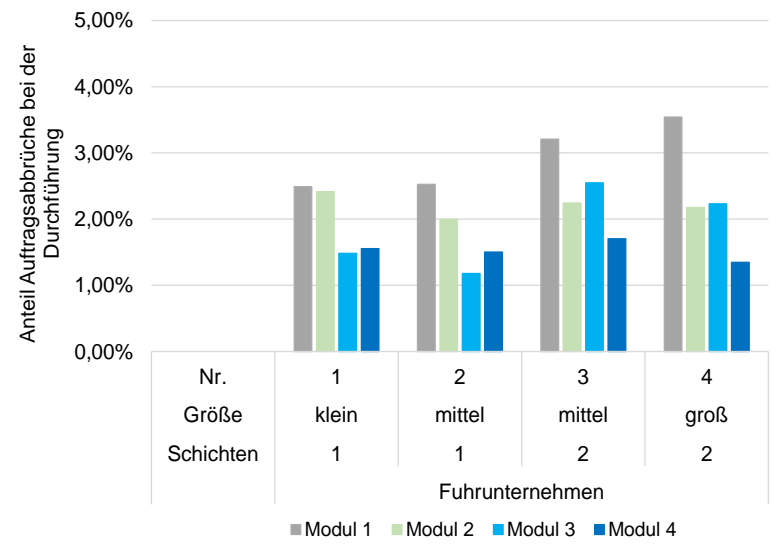

Abbildung 3: Anteil der Auftragsabbrüche bei der Durchführung

Der Anteil der Auftragsabbrüche ist bei zwei Schichten immer größer als bei einer Schicht, da dort beide Peaks des Verkehrs enthalten sind. Bei einer guten Verkehrssituation müssen weniger Aufträge abgebrochen werden, da auch bei der Zuweisung eines zeitlich kritischen Auftrages dieser mit einer höhren Wahrscheinlichkeit pünktlich durchgeführt werden kann. Bei einer schlechten Verkehrssituation müssen knapp zugewiesene Aufträge häufiger abgebrochen werden, da eine längere Fahrzeit eine Abfertigung verhindert. Innerhalb der zweiten Schicht liegen viele Schließzeiten der Logistikknoten, wodurch ein Abbruch des Auftrages nicht mehr nur an den Terminals, sondern auch an den Logistikknoten durch eine verspätete Ankunft möglich ist.

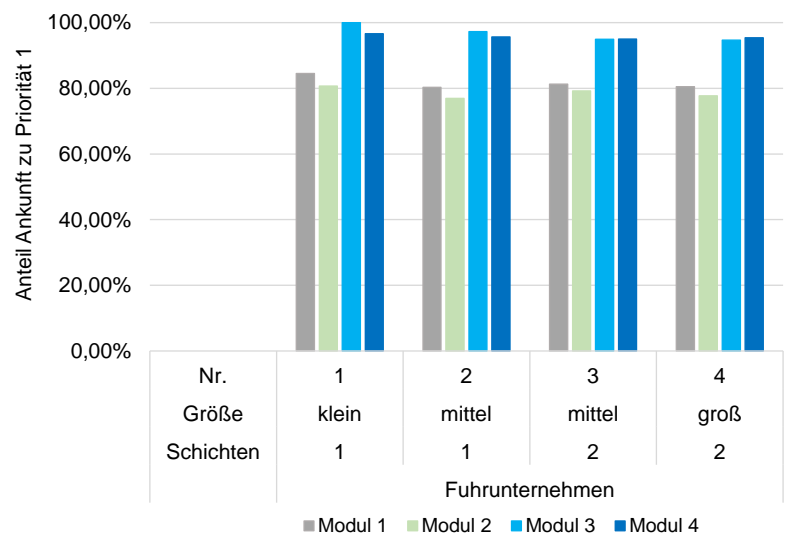

Abbildung 4: Anteil der Ankünfte innerhalb des Zeitfensters

Wie häufig die Lkw den Terminal innerhalb des Zeitkorridors mit der höchsten Abfertigungspriorität erreichen ist in Abbildung 4 dargestellt. Es ist ersichtlich, dass ein erlaubtes Umbuchen den Anteil der pünktlichen Ankünfte im Vergleich zum Grundmodell sowie Tauschmodell erhöht. Modul 2 führt zum geringsten Anteil der pünktlichen Ankünfte, wobei der Terminal häufig vor Zeitfensterbeginn erreicht wird. Eine Kombination der Möglichkeit des Umbuchens und Tauschens führt bei zwei Schichten zur Erhöhung der Pünktlichkeit, bei einer Schicht sinkt die Quote leicht. Sollte nur ein Umbuchen erlaubt sein, so erreichen Lkw des kleinen Unternehmens den Terminal am häufigsten pünktlich.

Die Auftragszuweisung des Simulationsmodells weist die Aufträge so zu, dass eine Ankunft am Terminal zu Priorität 2 nach dem Slot eher vermieden wird. Unabhängig von der Verkehrssituation wird bei erlaubter Umbuchung der Terminal häufiger innerhalb von Priorität 1 erreicht, ebenso wird die Ankunft zu Priorität 2 nach dem Slot vermieden. Das Tauschmodul führt tendenziell zu einer zu frühen Ankunft am Terminal. Die Verkehrssituation hat kaum Einfluss auf den Anteil der Priorität 1Ankünfte, allerdings wird der Terminal bei einer guten Verkehrssituation weniger häufig bzw. bei einer schlechten Verkehrssituation häufiger zu Priorität 2 nach dem Slot erreicht. Bei einem Auftragsabbruch an der Senke müsste der Lkw-Fahrer mit dem Container zum Depot fahren, um sein Chassis zu wechseln. Da dies vermieden werden soll, wird die Ankunft an der Senke mehrmals prognostiziert und der Auftrag im Simulationsmodell bereits an der Quelle abgebrochen, wenn ein Auftragsabbruch an der Senke wahrscheinlich ist. Die Senke wird unabhängig vom Fuhrunternehmen tendenziell eher zu früh erreicht. Bei erlaubtem Umbuchen wird die Senke fast immer innerhalb von Priorität 1 erreicht, da die Prognose häufiger vor Beginn des Zeitfensters durchgeführt wird, weshalb eine Umbuchung der Senke noch möglich ist.

Der Anteil umgebuchter Tourenpläne ist Abbildung 5 zu entnehmen.

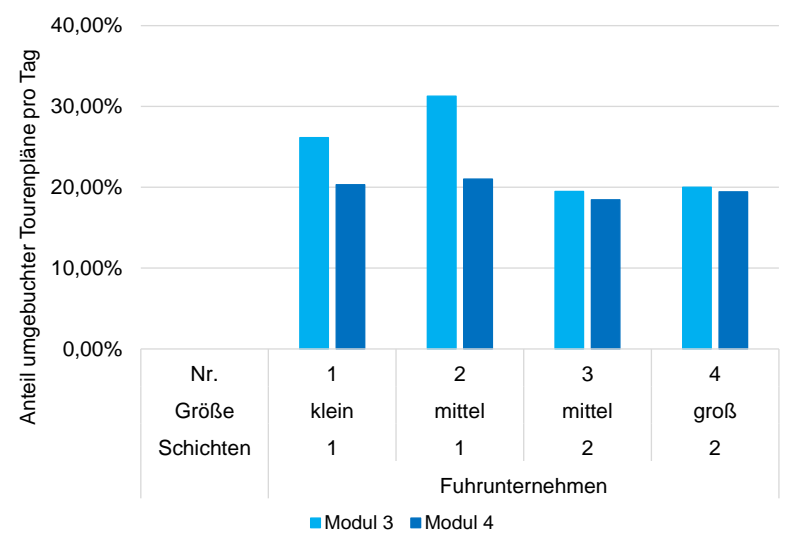

Abbildung 5: Anteil umgebuchter Tourenpläne pro Tag

Wenn nur Umbuchen erlaubt ist (Modul 3) ist der Anteil jeweils höher als bei dem kombinierten Modul 4. Bei einer Schicht sind weniger Restriktionen bei den Öffnungszeiten zu berücksichtigen, die eine Umbuchung teilweise verhindern. Daher buchen Unternehmen mit einer Schicht tendenziell häufiger um, wobei vor allem das 
mittlere Unternehmen mit einer Schicht oft umbucht.

Eine gute Verkehrssituation führt vermehrt, eine pessimistische Verkehrssituation hingegen zu weniger Umbuchungen. Die Veränderung bei Modul 3 ist größer als bei Modul 4. Bei gutem Verkehr werden $5 \%$ bis $10 \%$ mehr Tourenpläne umgebucht, da die Lkw zu früh ankommen würden. Bei schlechtem Verkehr wird weniger häufig umgebucht, da die Tourenplanung einen gewissen Puffer enthält.

\section{Fazit und Ausblick}

Die Studie hat gezeigt, dass die Möglichkeit des Stornierens und Umbuchens in TAS einen positiven Einfluss auf die Fuhrunternehmen hat und daher eine wichtige Flexibilitätsoption darstellt.

Dabei ist die Nutzung der Umbuchfunktion stärker von der Anzahl der Schichten als von der Unternehmensgröße abhängig. Nur Umbuchen führt bei kleinen Unternehmen nicht zwingend zu besseren Lösungen als nur Tauschen. Bei einer Kombination von Tauschen und Umbuchen muss seltener umgebucht werden und die Kombination der beiden Funktionen führt zu besseren Ergebnissen bei der AEQ als die Nutzung nur einer Flexibilitätsoption. Die Auswirkungen auf die AEQ fiel dennoch kleiner aus als erwartet.

Je mehr Flexibilitätsoptionen den Transportunternehmen zur Verfügung stehen, desto weniger Aufträge müssen abgebrochen werden. Zusätzlich konnte nachgewiesen werden, dass durch das Umbuchen die Wartezeit vor den Knoten sehr stark reduziert werden konnte und die Lkw sehr häufig während der Priorität 1-Abfertigung ankommen.

Eine besonders gute Verkehrssituation führt vermehrt zur Nutzung der Umbuchfunktion, eine besonders schlechte reduziert die Nutzung. Da die Auftragszuweisung tendenziell zu einer verfrühten Ankunft führt und genügend Puffer eingeplant wird, fallen die Auswirkungen einer durchschnittlich schlechten Verkehrssituation auf den kurzen Fahrstrecken nur wenig ins Gewicht. Neben den Vorteilen, die TAS für die Terminals haben, kann ein gut umgesetztes TAS auch Vorteile für das Fuhrunternehmen bieten, wenn er auch kurzfristig die vorhandenen Flexibilitätsoptionen nutzen kann und seine IT-Systeme so angepasst sind, dass er alle Funktionen des TAS richtig ausnutzen kann.

Die Arbeit konnte die vorangegangene Arbeit von Lange et al. [26] fortsetzen und das Modell realitätsnaher gestalten. Trotzdem mussten Annahmen getroffen werden, die teilweise von der Realität abweichen. Das Modell nutzt z. B. die vorhandenen Lenkzeiten der Fahrer nicht vollständig aus. Ebenso warten Fahrer teilweise bis zu eine Stunde am Tag auf die Zuweisung eines neuen Auftrages. Darüber hinaus ist anzumerken, dass die Daten nicht gezielt für die Simulationsstudie erhoben wurden, sondern angepasst und aufbereitet wurden.

In zukünftigen Arbeiten könnte untersucht werden, wie sich zusätzliche Terminvorgaben an den Logistikknoten auswirken. Bei Transportaufträgen, die zwei Slots benötigen, gilt es zu untersuchen, wie sich eine Dynamisierung, d. h. eine automatische Anpassung des Slots an der Senke bei Verzögerungen an der Quelle, auswirkt. Bei Transporten zwischen zwei Terminals sollte bei Slotverfügbarkeit an einem der beiden Terminals die Anlieferung oder Abholung am anderen Terminal garantiert werden können.

Darüber hinaus könnte untersucht werden, welche Auswirkungen eine Veränderung der Struktur des Transportaufkommens oder eine Verlängerung der Öffnungszeiten der Logistikknoten hat. Ein großes Einsparpotential liegt in der Verkürzung der Wartezeiten auf die Auftragszuweisung, hierfür könnte eine verbesserte Vorwegeplanung oder eine dynamischere Anpassung sowie Veränderungen von mehreren Slotzeiten implementiert werden.

Bisher wird eine veränderte Verkehrssituation nur bei der Durchführung berücksichtigt, womit eine Lastgrenze untersucht wird. Die veränderte Verkehrssituation könnte bereits bei der Auftragszuweisung berücksichtigt werden, um bessere Dispositionsentscheidungen treffen zu können. Bei der initialen Slotbuchung könnte versucht werden, unter der Voraussetzung, dass ausreichend Slots vorhanden sind, diese immer nur in direkt angrenzende Zeitfenster bei Transporten mit zwei Terminals zu buchen, um Wartezeiten und zu frühe Ankünfte an der Senke zu vermeiden.

\section{Literatur}

[1] DVV, "Mehr Umschlag, geringere Investitionen," URL: https://www.thb.info/rubriken/singleview/news/mehr-umschlag-geringere-investitionen.html [retrieved 5 July 2020].

[2] Ramírez-Nafarrate, A., González-Ramírez, R. G., Smith, N. R., Guerra-Olivares, R., and Voß, S., Impact on yard efficiency of a truck appointment system for a port terminal. Annals of Operations Research, Vol. 258, No. 2, 2017. pp. 195-216.

doi: 10.1007/s10479-016-2384-0.

[3] Dekker, R., van der Heide, S., van Asperen, E., and Ypsilantis, P., A chassis exchange terminal to reduce truck congestion at container terminals. Flexible Services and Manufacturing Journal, Vol. 25, No. 4, 2013. pp. 528542.

doi: 10.1007/s10696-012-9146-3. 
[4] Fan, Ren, Guo, and Li, Truck Scheduling Problem Considering Carbon Emissions under Truck Appointment System. Sustainability, Vol. 11, No. 22, 2019. p. 6256. doi: 10.3390/su11226256.

[5] Schulte, F., González, R. G., and Voß, S., Reducing Port-Related Truck Emissions: Coordinated Truck Appointments to Reduce Empty Truck Trips. Computational Logistics, edited by F. Corman, S. Voß and R. R. Negenborn, Springer International Publishing, Cham, 2015. pp. 495-509.

[6] Davies, P., Container terminal reservation systems. The 3 Rd Annual METRANS National Urban Freight Conference, 2009. URL: http://dtci.ca/wp-content/uploads/2011/10/Container-Reservation-Systems- [retrieved 1 July 2020].

[7] Huynh, N., Smith, D., and Harder, F., Truck Appointment Systems. Transportation Research Record: Journal of the Transportation Research Board, Vol. 2548, No. 1, 2016. pp. 1-9. doi: 10.3141/2548-01.

[8] Namboothiri, R., Planning Container Drayage Operations at Congested Seaports. Georgia Institute of Technology, 2006.

[9] Jula, H., Dessouky, M., Ioannou, P., and Chassiakos, A., Container movement by trucks in metropolitan networks: modeling and optimization. Transportation Research Part E: Logistics and Transportation Review, Vol. 41, No. 3, 2005. pp. 235-259. doi: 10.1016/j.tre.2004.03.003.

[10] Sterzik, S., and Kopfer, H., A Tabu Search Heuristic for the Inland Container Transportation Problem. Computers \& Operations Research, Vol. 40, No. 4, 2013. pp. 953-962. doi: 10.1016/j.cor.2012.11.015.

[11] Braekers, K., Ramaekers, K., and van Nieuwenhuys, I. e., The Vehicle Routing Problem: State of the Art Classification and Review. Computers \& Industrial Engineering, Vol. 99, 2015. URL: https://www.researchgate.net/publication/287796502_The_Vehicle_Routing_Problem_State_of_the_Art_Classification and Review.

[12] Yi, S., Scholz-Reiter, B., Kim, T., and Kim, K. H., Scheduling appointments for container truck arrivals considering their effects on congestion. Flexible Services and Manufacturing Journal, Vol. 16, No. 1, 2019. p. 87. doi: 10.1007/s10696-019-09333-y.

[13] Chen, G., Govindan, K., Yang, Z.-Z., Choi, T.-M., and Jiang, L., Terminal appointment system design by nonstationary $\mathrm{M}(\mathrm{t}) / \mathrm{Ek} / \mathrm{c}(\mathrm{t})$ queueing model and genetic algorithm. International Journal of Production Economics, Vol. 146, No. 2, 2013. pp. 694-703. doi: 10.1016/j.ijpe.2013.09.001.

[14] Torkjazi, M., Huynh, N., and Shiri, S., Truck appointment systems considering impact to drayage truck tours. Transportation Research Part E: Logistics and Transportation Review, Vol. 116, 2018. pp. 208-228. doi: 10.1016/j.tre.2018.06.003.

[15] Azab, A., Karam, A., and Eltawil, A., A Dynamic and Collaborative Truck Appointment Management System in Container Terminals. Proceedings of the 6th International Conference on Operations Research and Enterprise Systems, SCITEPRESS - Science and Technology Publications, 2017. pp. 85-95.
[16] Phan, M.-H., and Kim, K. H., Negotiating truck arrival times among trucking companies and a container terminal. Transportation Research Part E: Logistics and Transportation Review, Vol. 75, 2015. pp. 132-144. doi: 10.1016/j.tre.2015.01.004.

[17] Zehendner, E., and Feillet, D., Benefits of a truck appointment system on the service quality of inland transport modes at a multimodal container terminal. European Journal of Operational Research, Vol. 235, No. 2, 2014. pp. 461-469.

doi: 10.1016/j.ejor.2013.07.005

[18] Namboothiri, R., and Erera, A. L., Planning local container drayage operations given a port access appointment system. Transportation Research Part E: Logistics and Transportation Review, Vol. 44, No. 2, 2008. pp. 185-202. doi: 10.1016/j.tre.2007.07.004.

[19] Shiri, S., and Huynh, N., Optimization of drayage operations with time-window constraints. International Journal of Production Economics, Vol. 176, 2016. pp. 7-20.

doi: 10.1016/j.ijpe.2016.03.005.

[20] Huynh, N., Reducing Truck Turn Times at Marine Terminals with Appointment Scheduling. Transportation Research Record: Journal of the Transportation Research Board, Vol. 2100, No. 1, 2009. pp. 47-57. doi: 10.3141/2100-06.

[21] Do, N. A. D., Nielsen, I. E., Chen, G., and Nielsen, P., A simulation-based genetic algorithm approach for reducing emissions from import container pick-up operation at container terminal. Annals of Operations Research, Vol. 242, No. 2, 2016. pp. 285-301. doi: 10.1007/s10479-014-1636-0.

[22] Huynh, N., [dissertation] Methodologies for reducing truck turn time at marine container terminals. 2005.

[23] Huynh, N., and Walton, C. M. (eds.), Improving Efficiency of Drayage Operations at Seaport Container Terminals Through the Use of an Appointment System, 2011.

[24] van Asperen, E., Borgman, B., and Dekker, R., Evaluating impact of truck announcements on container stacking efficiency. Flexible Services and Manufacturing Journal, Vol. 25, No. 4, 2013. pp. 543-556. doi: 10.1007/s10696-011-9108-1.

[25] Speer, U., Optimierung von automatischen Lagerkransystemen auf Containerterminals, Springer Fachmedien Wiesbaden, Wiesbaden, 2017.

[26] Lange, A.-K., Grafelmann, M., Schwientek, A., and Jahn, C., Effizientes Tauschen der Zeitfenster von Transportaufträgen in Truck Appointment Systems: Efficient Swapping of Time Windows of Transport Orders in Truck Appointment Systems. $1^{\text {st }}$ ed., edited by M. Putz and A. Schlegel, Wissenschaftliche Scripten, Auerbach /Vogtl., 2019. pp. 295-304.

[27] Arnold, D., Isermann, H., Kuhn, A., Tempelmeier, H., and Furmans, K. (eds.), Handbuch Logistik, $3^{\text {rd }}$ ed., Springer Berlin Heidelberg, Berlin, Heidelberg, 2008. 